\title{
A REVIEW ON THE ROLE OF NANOCRYSTALS AND NANOSUSPENSIONS IN DRUG DELIVERY SYSTEMS
}

\section{S. J. SHANKAR ${ }^{1 *}$, JASWANTH GOWDA B. H. ${ }^{2}$, AKSHATHA R. S. ${ }^{3}$, BASAVARAJ METIKURKI ${ }^{4}$, MOHAMED REHAMATHULLA ${ }^{5}$}

1,2Department of Pharmaceutics, Vivekananda College of Pharmacy, Dr. Rajkumar Road, Rajajinagar $2^{\text {nd }}$ Stage, Bengaluru 560055, ${ }^{3}$ Department of Pharmaceutics, PES College of Pharmacy, PES University, 50 Feet Road, Hanumanthnagar, Bengaluru 560050, ${ }^{4}$ Department of Pharmaceutical Chemistry, Nitte College of Pharmaceutical Sciences, NMIT Campus, Yelahanka, Bengaluru 560064, ${ }^{5}$ Department of Pharmaceutics, College of Pharmacy, King Khalid University, Abha, Asir Provience, Saudi Arabia, Post Box No-960 Email: sjjyothi@gmail.com

Received: 28 Aug 2019, Revised and Accepted: 08 Nov 2019

\section{ABSTRACT}

Nearly $40 \%$ of drugs coming to the market nowadays are having poor solvency related issues and $70 \%$ molecules in the discovery pipeline are in effect fundamentally insoluble in water. Nanocrystals is an unmistakable instrument to tackle the issue identified with poor fluid solvency and helps in improving the bioavailability of various drugs as presented in the literature. The particle size reduction came about into the temperamental nanocrystalline system and the phenomenon of ostawald ripening happens. These techniques are preparing to the improvement of nanosized objects, which can play out multiple technological tasks. There are a few couples of noteworthy benefits of nanocrystal formulations, for example, upgrade oral bioavailability, improved dose proportionality, reduced food effects, appropriateness for administration by all routes and probability of sterile filtration because of diminished particle size range. One of the most adequate preferences of nanocrystals is their wide scope of utilization, for example, ophthalmic delivery, oral delivery, transdermal delivery, pulmonary delivery, intravenous delivery and targeted delivery, especially for tumour and brain. The increment in the commercial value of nanocrystals just as the measure of nanocrystal products in the market is picking up more of attention to be utilized as a strategy so as to get commercial advantages. In this paper, a brief and accurate precis of nanosuspension is stated with a specific spotlight on nanosuspension preparation methodologies, benefits and few major applications of nanosuspensions.

Keywords: Nanocrystal, Nanosuspension, Bioavailability, Solubility, Media milling, Dissolution rate, Bottom-up approach, High-pressure homogenization

(C) 2020 The Authors. Published by Innovare Academic Sciences Pvt Ltd. This is an open-access article under the CC BY license (http://creativecommons.org/licenses/by/4.0/] DOI: http://dx.doi.org/10.22159/ijap.2020v12i1.35508. Journal homepage: https://innovareacademics.in/journals/index.php/ijap

\section{INTRODUCTION}

Nanotechnology has prompted the rise of another field called nanomedicine, which includes the utilization of nanotechnology in drug development, improves and offers many all the more energizing guarantees of new diagnoses and cures [1]. A newer approach for drug delivery for poorly water-soluble compounds has gained lot of attention. Where in the drug is presented in the form of nanometersized particles/crystals that can be dispersed in a colloidal solution by having a stabilizer on the surface of nanoparticles. Nano sizing alludes to the way toward diminishing the size of active pharmaceutical ingredient (API) to the submicron level. Nanosuspensions are aqueous dispersions comprising of a blend of API and stabilizers, for example, surfactant or polymer in water. These stabilizers help in avoiding the clump of nanoparticles and keep them separate from one another by giving electrostatic/steric repulsion on the surface of nanoparticles. Nanotechnology is characterized as the Science and Engineering done in the nanoscale that is $10-9 \mathrm{~m}^{2}$. The nanoparticles are typically made utilizing the media milling method. The shear forces involved during the impaction of the milling procedure on the micron size drug crystals brings about nanometer-sized particles of the drug that can be dispersed in water and stabilized by the surface ligands acquainted with their surface [2]. Most extensively, nanomedicine is the way toward diagnosing, treating, preventing disease and traumatic injury, relieving pain and safeguarding in improving human health, utilizing molecular-level knowledge of the human body [3].

Nanosuspensions have been prescribed as an all-inclusive delivery approach for orally administered drug particles that fall into BCS class II and class IV category of drugs [4]. Butler and Dressman proposed the developability classification system (DCS) as a way to divide compounds in a more biorelevant way [5]. As per the DCS, which shows contrasts between dissolution rate-limited and solubility limited compounds, the intrinsic solubility and the related intraluminal drug concentration for compounds comes under class II and IV are too low to even consider achieving adequate flux over the epithelial membrane. Subsequently, complexation or formulation approaches lean on solid-state modifications that might be best appeared differently in relation to nanocrystals for compounds that comes under DCS Class II b and IV $[6,7]$. Nowadays, the new chemical entities coming out of discovery laboratories in the pharmaceutical industry habitually have low water dissolvability because of the high throughput screening process [8,9]. Various formulation techniques have been created to address the low fluid solvency of pharmaceutical compounds [10]. Since, particle size reduction (i.e., via micronization) is a most usual technique that has been utilized as a means for upgradation of the absorption of least dissolvable drugs via oral route. In this technique, particle size is reduced to enhance the performance of food, drug, and spices. Although, the degree of dissolution rate upgradation is highly needed to enhance the in vivo drug absorption for poorly soluble drug applicants are more prominent than that which can be given by micronization [11]. Thus, the further reduction in particle size of compounds to the range of nanometer must be considered as a strategy for up-gradation of the absorption of these drugs via oral route [12]. Numerous reports which have published recently have shown that the nanoparticles have the capabilities in upgradation of the oral absorption of poorly soluble drugs along with the betterment of other biopharmaceutical properties too (e. g., variable absorption in the fed vs. fasted state) [13-15].

Recent studies on solvency and dissolution improvement after nanosizing have reported variable results $[16,17]$. The most widely recognized practice for the solubility assurance detailed depended on the separation by filtration to isolate non-dissolved nanoparticles from the solution utilizing the membrane filters of different pore sizes $(0.1$, 0.22 , and $0.45 \mathrm{~m}$ pore size) [18-20]. A couple of reports have likewise demonstrated the utilization of molecular weight cut-off filters [21], dialysis bag method [22], centrifugation [23], and ultracentrifugation [24] for the separation of non-dissolved and dissolved nanocrystals while estimating solubility. Although, proof of adequate separation of non-dissolved and dissolved nanocrystals has been unclear. Determining precise solubility is a genuinely necessary instrument in the pre-formulation examination of any nanocrystalline particles of a poorly soluble compound. Nevertheless, the accurate measurement of 
solvency during the drug molecule in the range of nanometer is a great challenge. Complicating this is the inclination of nanoparticles that stay suspended in the solution even after centrifugation [25]. Accordingly, the development of a technique for accurate solubility determination related with nanocrystals is one of the aims for current researchers.

One of major disadvantage of nanocrystals includes instability during storage. Ostwald ripening alludes to a phenomenon that small particles steadily solubilise and settle on the larger particle surface after some time. It happens when the particle size in a dispersion system is heterogeneous and the dispersed phase has a limited solubility in the aqueous mediums, which, tragically, are the conditions much of the time experienced in pharmaceutical suspension. Ostwald ripening prompts the particle size development and physical instability of a dispersion system while storage; in this way, there is a solid requirement for anticipating this process. Surfactants and polymers are regularly utilized as stabilizers to defer separation and connection of drug molecules at the surface of dispersed particles. Polymers are accepted to be more powerful than little molecular weight surfactants since they will, in general, adhere to the nanocrystal surface less dynamically than surfactants. Another method for avoiding particle size growth is to formulate uniform nanocrystals, along these lines disposing of one of the conditions for Ostwald ripening. Advancing the process parameters, for example, a number of high-pressure homogenization cycles, speed of milling and time of milling can dispose of huge particles and acquire size distribution of narrow range. Handling nanosuspensions into solid products is another method of preventing dynamic changes in the medium and in this manner, Ostwald ripening can we eliminated [26]. The search criteria used for review of this paper were nanocrystals, nanosuspensions in various platforms like Pubmed, Science Direct, Scopus and Innovare Academic Sciences Etc.,

The main aim of this paper is to furnish a brief overview on nanosuspension, it is expected that this review article will give coherent information on nanosuspension and stand as a starting step for further advancement of nanosuspension.

\section{Nanosuspensions}

Nanosuspensions are submicron colloidal scatterings of nanosized drug particles that are stabilized by surfactants [27]. Nanosuspension is helpful in enhancement of dissolvability of drugs that are least soluble in water and lipid media. This philosophy is important for molecules with poor solubility, poor permeability or both which represents a huge test for the formulators [28]. Nanosuspensions are liquid dispersion which contains solid drug nanoparticles that are stabilized by surfactant and/or polymer [29].

A nanosuspension not just illuminates the issues of least dissolvability and bioavailability yet, in addition, modifies the drug pharmacokinetics and that improves safety and viability of drugs. If there should arise an occurrence of drugs that are insoluble in both water and inorganic media as opposed to utilizing lipoid systems, nanosuspensions are utilized as a formulation strategy. Nanosuspension formulation strategy is most suitable for the molecules with high $\log \mathrm{P}$ value, large dose and high melting point. The usage of nanotechnology to formulate least water dissolvable drugs as nanosuspension offers the opportunity to address the nature of the deficiency related with this class of drugs. Nanosuspension has been accounted to improve absorption and bioavailability, it may diminish the dose of the conventional oral dosage forms. Drug particle size reduction prompts an expansion in surface area and therefore in the rate of dissolution as portrayed by the Nernst-Brunner and Levich alteration of the Noyes-Whitney equation. Besides, enhancement in saturation dissolvability is proposed by particle size reduction because of an expanded dissolution pressure clarified by the Ostwald-Freundlich equation. An enhanced measure of amorphous drug fraction could initiate higher saturation dissolvability. In nanosuspension technology, the drug is kept up in the required crystalline state with decreased particle size, prompting an enhanced dissolution rate and as needs be improved bioavailability. Medications enclosed inside nanosuspensions exist in the pharmaceutically satisfactory crystalline or amorphous state [30]. There are two primary methodologies for the formulation of nanosuspension that are a bottom-up and top-down strategy. The bottom-up strategy involves solubilizing drug in a solvent and further addition of non-solvent to precipitate crystals. The top-down strategy depends on mechanical rubbing to furnish huge crystalline particles size less than about 400 $\mathrm{nm}$ are justifiable for administration [29].

\section{Benefits of nanosuspension technology for poorly soluble drugs}

1) Diminished particle size, enhanced drug dissolution rate, enhanced rate and extent of absorption, enhanced bioavailability of the drug, area under plasma versus time curve, peak drug level, onset of time, decreased variability and lowered fed/fasted impacts.

2) Compounds that are insoluble in water and soluble in oil can be formulated as nanosuspension, then again, nanosuspension can be utilized with lipoid systems and effectively formulate compounds that are insoluble in both water and oil.

3) Nanoparticles prolongs the contact time of drug by adhering on to the gastrointestinal mucosa thus increase its absorption.

4) Nanosuspension has got many routes of administration such as parenteral, oral, dermal, ocular and pulmonary makes it most convenient.

5) Nanosuspension of nanoparticles offers different favorable circumstances over conventional ocular dosage forms, incorporating lower in the measure of dose, upkeep of drug release over a delayed timeframe, decrease in systemic toxicity of drug, improved drug absorption because of longer residing time of nanoparticles on the surface of cornea, higher concentration of drug in the infected tissue, and suitability for inadequately water-soluble drugs, and smaller particles are preferred endured by patients over bigger particles, in this way, nanoparticles may speak to promising drug carriers for ophthalmic applications.

6) Nanosuspension possess the least incidence of adverse reactions by the excipients.

7) Nanosuspensions defeat delivery issues for the compounds by deterring the need to dissolve them and by keeping up the drug in a favoured crystalline state of size adequately little for pharmaceutical acceptability.

8) Nanosuspension has got resistance to hydrolysis, oxidation and also posses' increased physical stability to settling.

9) Decreased administration quantities need for intramuscular, subcutaneous, and ophthalmic use.

10) Last but not least, nanosuspensions can help out in passive targeting [31-33].

\section{Formulation approaches of nanosuspension}

There are two strategies for the manufacturing of nanosuspensions, "Bottom-Up" and the "Top-Down" technologies. Traditional techniques for precipitation are classified "Base Up Technology". The "Top-Down Technologies" are the disintegration methods and are favoured over the precipitation methods. This incorporates High-Pressure Homogenization in water, Media Milling, HighPressure Homogenization in nonaqueous media finally, blends of Precipitation and High-Pressure Homogenization.

The top-down technique can be further divided into two approaches homogenization and attrition wet media milling.

\section{Attrition wet media milling}

An active drug substance is scattered with an aqueous solution in which the stabilizers were pre-isolated, the surface of nanocrystals is exceptionally strong and has high surface vitality, and it should be stabilized by a single or mixture of stabilizers. Stabilizers can be ionic or stearic and can be used as a solitary and/or in a combination of polymeric as well as surfactant stabilizers. At that point, the solution is poured in the grinding chamber alongside spherical beads and balls while the globules are pivoted at rapid. It is believed that because of the attrition between molecules' surface and the surface of the globule, particle size reduction occurs; the globules 
and balls serving as a milling media. Globules are available in different sizes and are of different materials, however, for the most part, are made of glass, zirconium oxide, or polymeric material. The sort of material the globules are made of is a critical factor as they can interface with the dynamic drug substance. Albeit costly, these Beads are the best option in contrast to keeping away from impurities in the last formulation [36]. The size of the beads has a direct relationship with the ideal range of particle size in the formulation of nanocrystals [37]. The standard length for conventional milling utilizing overhead stirring is somewhere close to $3 \mathrm{~h}$ and $12 \mathrm{~h}$. Doubtlessly, these parameters can change from molecule to molecule. Milling should be stopped once the desired particle size range is achieved. The rotational speed of the milling media is too critical parameter. With the too moderate speed, the globules can't pivot proficiently and milling can't be performed precisely, and with the too quick speed, the equally rotating balls may stay at the upper surface of the media and milling is halted. With a systematic study by trial and error the formulator selects the stabilizers, as well as other milling parameters and optimizes them in order to achieve the required particle size range and stability. The final product characteristics can vary, depending on the amount of globules, the ratio of active drug substance to the amount of globules, the ratio of concentrations of active substance to the stabilizer, milling time, milling temperature as well as milling duration [38]. This technique is simple, inexpensive, and easily scalable. The only drawback associated with this technology is the contamination related to the beading material. That aside, several products have successfully reached the commercial level using this technology. Djordje Medarevic et al. formulated carvedilol nanosuspension by using wet media milling with help of hydroxyl propyl cellulose SL and sodium lauryl sulphate as stabilizers that resulted in stabilized formulation with enhanced dissolution rate [39]. Ligang Guo et al. used wet media milling to prepare andrographolide nanosuspension with non-ionic and ionic stabilizers resulted in nanosuspension having a particle size of about $300 \mathrm{~nm}$ with no chemical degradation [40]. Chengying Shen et al. has prepared nitrofurazone nanosuspension by using wet media milling has shown better bioavailability via dermal route when compared to nitrofurazone marketed gel [41]. Song Huang et al. used wet milling to formulate nanosuspensions of efonidipine hydrochloride resulted in enhanced bioavailability [42].

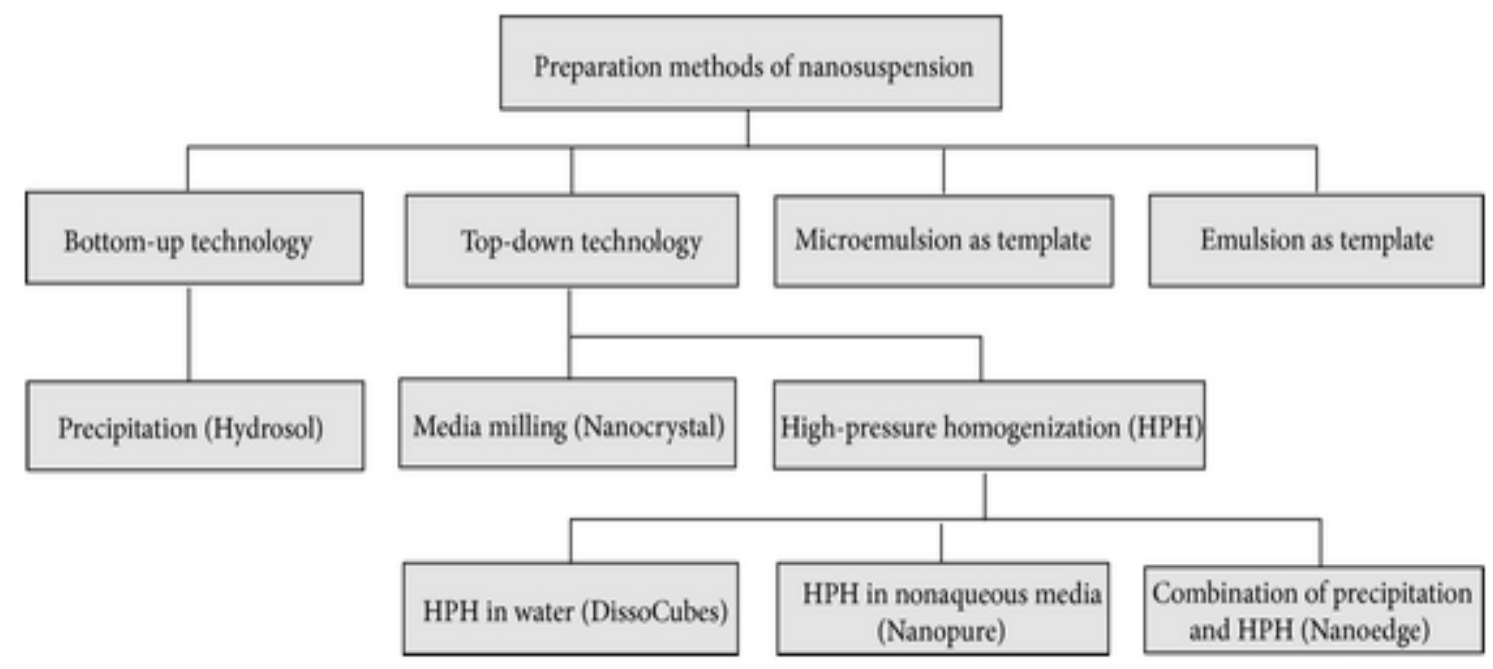

Fig. 1: Schematic diagram of preparation methods of nanosuspension [34]

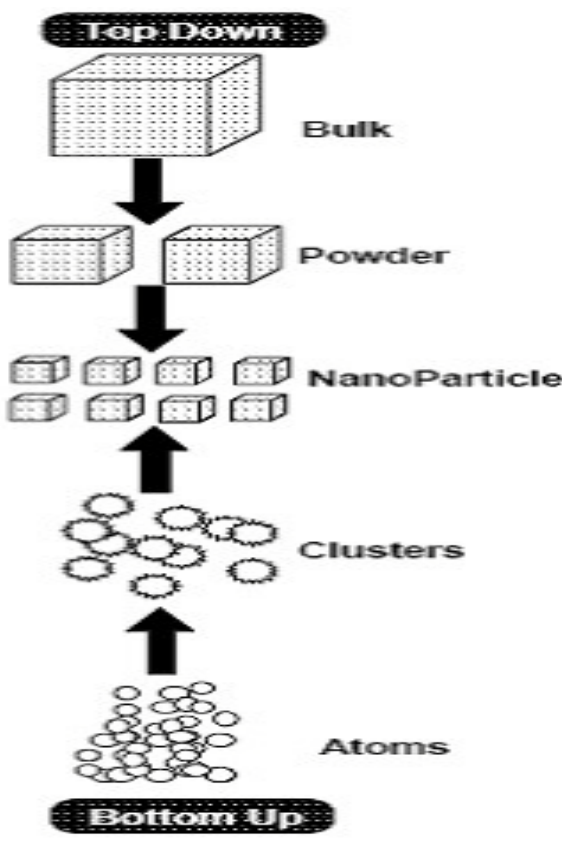

Fig. 2: Top-down and bottom-up approach [35] 


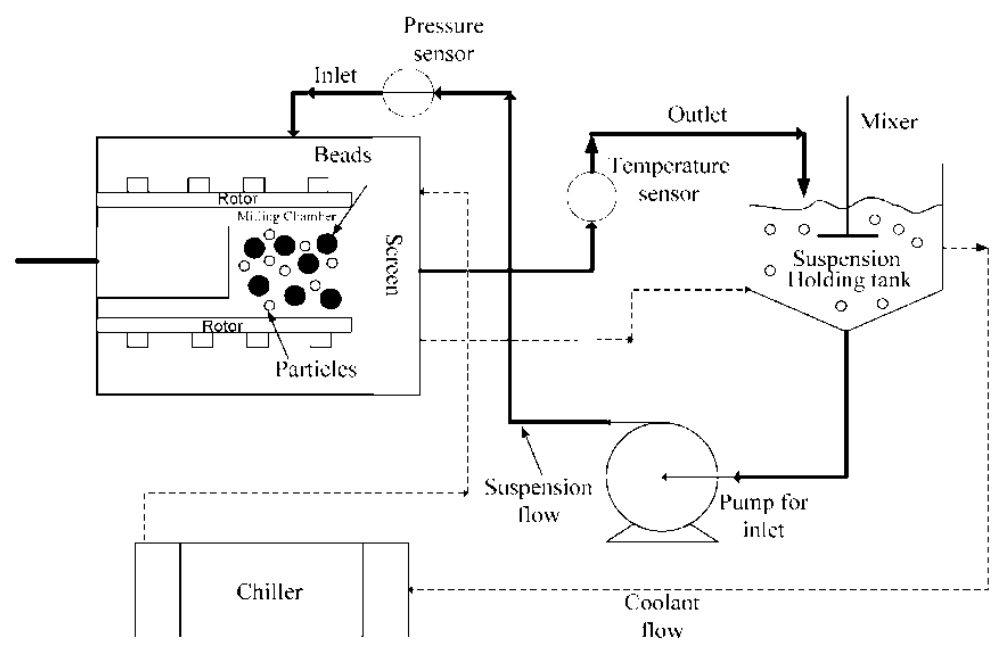

Fig. 3: Schematic representation of attrition wet media milling [43]

\section{High-pressure homogenization}

The fundamental guideline is high pressure that is 100-1500 bars. By this pressure, we can undoubtedly change over the micron size particle, into nanosize particle. Moreover, it at first needs the micron range particle that is $<25$ micrometer, with the goal that we need to get the example from the jet mill on the grounds that by utilizing jet mill we can decrease the particle size up to $<25$ micrometers. Furthermore, we can use the equipment for both batch and continuous operations. Capacity is also $40 \mathrm{ml}$ to thousand litres. Here first, we need to change over the particles into the form of presuspension. High pressure and high shear are because of collision of particles, the size of the particle will be diminished. Here, we need to add viscosity enhancers to build the viscosity of nanosuspension. In this method we need to majorly focus on the two parameters called pressure and homogenization cycles [44]. Hui wang et al. has formulated daidzein nanosuspension via high-pressure homogenization technique by using stearic and electrostatic stabilizers to have improved solubility and oral bioavailability [45]. Alptug Karakucuk et al. has prepared Ritonavir nanosuspension via high-pressure homogenization using microfluidizer with HPMC 3 cps and sodium dodecyl sulfate as stabilizers resulted in improved oral bioavailability in fed state [46]. Emine Tashan et al. formulated the nanosuspension of ziprasidone by using microfluidizer resulted in increased aqueous solubility compared to a coarse powder and physical mixtures of drug [47]. Sumathi R et al. prepared polymeric nanosuspension of naringenin by utilizing high-pressure homogenization leaded in elevated dissolution rate and better stability [48].

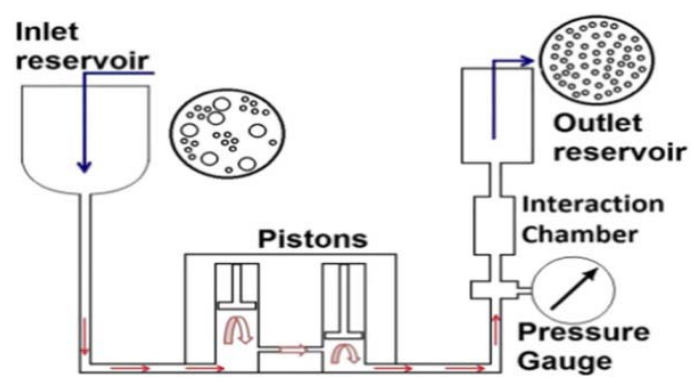

Fig. 4: Schematic representation of high pressure homogenization [49]

\section{Bottom up approach}

Bottom up methodology is otherwise called precipitation technique. Precipitation has been connected for quite a long time to form submicron particles inside the most recent decade, particularly for the poorly soluble drugs. Normally, the drug is dissolved in a solvent firstly. At that point, this solution is blended with a miscible antisolvent with the help of surfactants. Fast addition of a drug solution to the anti-solvent (typically water) prompts unexpected supersaturation of the drug in the blended solution and formation of ultrafine crystalline or amorphous drug solids. When setting up a stable suspension with the least particle size, a high nucleation rate however low growth rate is fundamental. The two rates are reliant majorly on temperature, where the optimum temperature for nucleation may lie beneath that for growth of crystal, which further permits temperature optimization [30]. Harsha Kathpalia et al. have prepared atovaquone nanosuspension by anti-solvent precipitation and $\mathrm{pH}$ based precipitation methods with the help of poloxamer, povidone and phospholipids that resulted in not only stability of suspension but also the dissolution rate of nanosuspension [50]. Aline Martins dos Santos et al. were used nanoprecipitation technique to obtain methotrexate nanosuspension which resulted in improved dissolution rate of methotrexate [51]. Mohammad $\mathrm{H}$. Shariare et al. used the anti-solvent precipitation technique for the preparation of furosemide nanosuspension has increased relative bioavailability of about 2.3 folds than pure drug [52]. HE Suna et al. used an anti-solvent sonication-precipitation technique to prepare teniposide nanosuspension by using PVP as a stabilizer which resulted in physically and chemically stable suspension for atleast $10 \mathrm{~d}$ at $4^{\circ} \mathrm{C}$ [53]. Bibaswan Mishra et al. were used controlled precipitation ultrasonication method to prepare nanosuspensions of naproxen which has helped to overcome the problems of bioavailability [54] Manishaanjane et al. prepared valsartan nanosuspension with help of nanoprecipitation technique showed better stability and elevated solubility [55]. Dekate $\mathrm{S}$ et al. used precipitation techniques to formulate curcumin nanosuspension that resulted in better in vitro dissolution rate than plain curcumin via oral route [56].

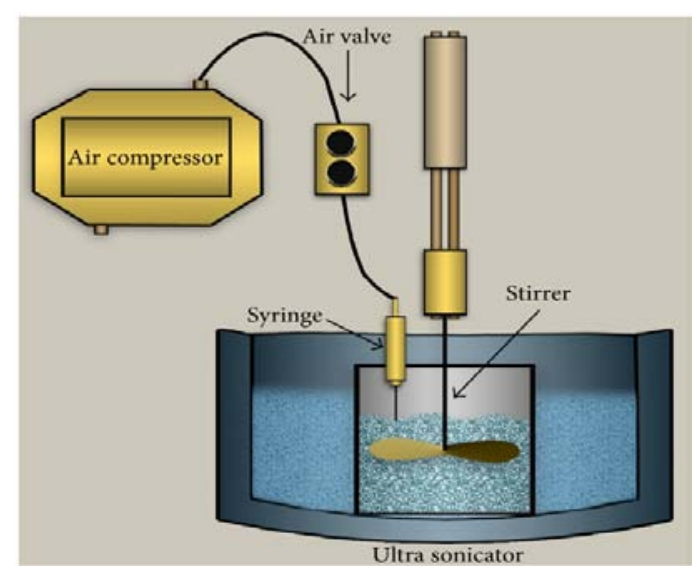

Fig. 5: Schematic representation of the precipitation method [34] 


\section{Emulsion diffusion method}

The emulsion can also be utilized as templates to create nanosuspension apart from drug delivery vehicles. The Drugs that are soluble in either or partially water-miscible solvent or volatile organic solvent can utilize the emulsions as a template. Such solvents can be utilized as the dispersed phase of the emulsion. Mixture of organic solvents stacked with the drug is dispersed in the aqueous phase containing appropriate surfactants along with stirring to result in an emulsion. The formed emulsion was additionally homogenized by high-pressure homogenization. After homogenization cycles, water has been added to dilute the emulsion, homogenized to diffuse organic solvent and convert the droplets into the solid particles. Since one particle is shaped in every emulsion droplet, it is conceivable to control the particle size of the nanosuspension by controlling the size of the emulsion optimizing the composition of surfactant which enhances the intake of organic phase and eventually loading of drug in the emulsion. Fundamentally methanol, ethanol, ethyl acetate chloroform are utilized as organic solvents [57-60].

\section{Micro emulsion template}

This technique pursues mixture solvent or organic solvent loaded with the drug dispersed in an aqueous phase which contains an appropriate surfactant to result an emulsion. Further the organic phase was evaporated under the reduced pressure to make that the particles of drug precipitate quickly to give the nanosuspension which is later on stabilized by surfactants. Another method utilizes partially water miscible solvents, for example, butyl lactate, benzyl alcohol and triacetin as the dispersed phase rather than dangerous solvents [61].

\section{Combination approach}

Combination strategy was created to defeat the issues of the top down and bottom up techniques. This technique is the combination of top down and bottom up techniques. This technology is the two stage process. Initial step is the pre-treatment process (bottom up) and second step is reduction of the particle size. In second step, high pressure homogenization is utilized. This procedure is also known as annealing. Annealing is the way toward converting the unstable matter into the stable form by single or rehashed use of energy, trailed by thermal relaxation. The nanocrystals formed utilizing combination technology has particle size underneath $200 \mathrm{~nm}$.

\section{Applications of nanosuspension}

The applications of nanosuspensions had land marking history. Among these few applications are given below.

\section{Oral drug delivery}

Inadequate solubility, lacking disintegration, and deficient efficacy are the serious issue of oral route of drug administration. Because least size of particles and higher surface to volume ratio, oral nanosuspensions are remarkably used to build the rate of absorption and bioavailability of least dissolvable drugs [62]. If there should be an occurrence of azithromycin nanosuspensions, over $65 \%$ of medication was seen to be dissolved in $5 \mathrm{~h}$ as appeared differently in relation to $20 \%$ of micronized drugs [63]. The nanosuspensions have major involvement in improved oral absorption, dose proportionality, and low inter subjected variability. By using standard manufacturing strategies, drug nanosuspensions can be basically joined into different dosage forms like fast melts, capsules and tablets. The nanosuspension of ketoprofen was adequately consolidated into pellets for the sustained release of the drug over the time of $24 \mathrm{~h}$ [64].

\section{Parental drug delivery}

The present methodologies for parental delivery loaded micellar solutions, salt formation, solubilisation using co-solvents, cyclodextrin complexation, and all the more as of late vesicular frameworks for instance, liposomes and niosomes. In any case, these strategies have constrainments like solubilisation limit, parental acceptability, high manufacturing cost, to solve the above problems, the nanosuspension technology is used. nanosuspensions are administered through different parental routes, for example, intra-articular, intraperitoneal, intravenous, and so on also, nanosuspensions increment the viability of parenterally administered drugs. Paclitaxel nanosuspension was reported to have their predominance in diminishing the median tumour burden [2]. Clofazimine nanosuspension exhibited advancement in stability and also efficacy above the liposomal clofazimine in mycobacterium avium-infected female mice [65]. Rainbow et al. showed that intravenous nanosuspension of itraconazole enhanced the adequacy of antifungal action in rats relative to the solution formulation [66].

\section{Pulmonary drug delivery}

For delivering via pulmonary, nanosuspensions can be nebulized through mechanical or ultrasonic nebulizers. Inside seeing fine particles, all aerosol droplets contain medicated nanoparticles. Budesonide Corticosteroid has been viably formulated as nanosuspension for delivery via pulmonary route [67]. Aqueous suspensions of the medication can be effectively nebulized and directed by pulmonary route as the particle size is practically low. Different sorts of nebulizers are accessible for the administration of liquid formulations. There are few deugs that have been effectively attempted with pulmonary route are ibuprofen, ketotifen, indomethacin, budesonide, doxorubicin, nifedipine, interleukin-2, itraconazole, p53 gene, leuprolide, etc [68].

\section{Ocular drug delivery}

The tear fluid secreted from lachrymal gland has least drug dissolving capabilities. On the off chance that it is planned as nanoparticles its dissolvability and bioavailability will elevate [69]. Nanosuspension is used to deliver drugs via ocular route for the purpose of sustained release. Liang and co-workers formulated cloricromene nanosuspension for ocular delivery with help of eudragit. Examination indicated higher availability of drug in aqueous humor of rabbit eye. As such, nanosuspension offers a promising strategy for improving the bioavailability and shelf-life of the drug after the ophthalmic application [70].

\section{Targeted drug delivery}

Nanosuspensions are suitable for targeting specific organs in perspective on their surface properties. Close by this, it is anything but difficult to adjust in vivo conduct by changing the stabilizer. The drug will be taken up by the mononuclear phagocytic system which allows region-specific delivery of the drug. This can be used for concentrating on anti-fungal, anti-mycobacterial, or anti-leishmanial drugs to macrophages if the pathogens suffer intracellularly [71]. Kayser prepared an aphidicolin nanosuspension that improved the targeting of drug to macrophages which were infected by leishmania. He expressed that the drug as nanosuspension had ec50 of $0.003 \mu \mathrm{g} / \mathrm{ml}$, while the conventional form had $0.16 \mu \mathrm{g} / \mathrm{ml}$ [72]. Scholer et al. depicted an increased targeting of drug to the brain in the treatment of toxoplasmic encephalitis by utilizing an atovaquone nanosuspension [73].

\section{Transdermal drug delivery}

Nanocrystals are evolved greatly in the field of transdermal delivery of the drug to overcome the solubility issues of drugs, in this manner expanding the concentration gradient in between the formulation and the skin, subsequently, transdermal permeation of the drug [26]. Due to its nano size in nature it exhibit better permeability than microsized particles. A transient stability test demonstrated that all nanosuspension remained sensibly stable at various temperatures. The Lutein nanocrystals had the option to penetrate through cellulose nitrate layers, utilized as an in vitro model of a penetration barrier, 14 times superior to coarse powder. In any case, no permeation through pig ear skin was watched, which shows that the Lutein entering the skin stayed there because of the lipophilicity [74].

\section{Mucoadhesion of the nanoparticles}

A nanoparticle has got capabilities to adhere on the mucosal surface because of tiny nano sized particles. During initial stages adhesion of particle occur followed by its absorption. To elevate much higher contact period nanosuspension is formulated along hydrogels made from variety of mucoadhesive polymers. The adhesiveness of the nanosuspension enhances the bioavailability as well as improves targeting of the parasites continuing in the GIT. Bupravaquone Mucoadhesive Nanosuspensions have been accounted for to exhibit a favorable position in TRC Alpha Deficient mice tainted with Cryptosporidium Parvum Oocytes [75]. 


\section{Future perspectives}

While the plan of nanoparticulate formulations has turned out to be progressively refined, fundamentally and reasonably basic nanocrystals have a one of a kind favorable position as for the advancement of marketed products. Nanocrystals can enormously upgrade the saturation dissolvability and dissolution rate of least dissolvable drugs with basic technologies for production and components. The huge contact area of nanocrystals can take into consideration a more noteworthy communication with tissue or cell surfaces and upgrade drug absorption. A few oral nanocrystal products are accessible on the market and dermal and IV products are effectively investigated. Notwithstanding, the capability of nanocrystals has not been completely examined for various applications, for example, targeted or local drug delivery. Attachment with a ligand introduces a chance to convey nanocrystals in a target-specific manner. Notwithstanding, the present methods of nanocrystal production and alteration don't satisfactorily address the difficulties being developed of such products, and new ways to deal with engineer nanocrystals are unequivocally anticipated.

\section{CONCLUSION}

Nanocrystals can be adopted by all poorly soluble drugs to defeat their solubility and bioavailability issues. The reduction in particle size to nanometer range adds to the enhanced particle surface, curvature, saturation solubility, dissolution velocity and further reasonable bioavailability. Nanosuspensions are specific and economically conceivable way to deal with tackle the issues of hydrophobic drug, for example, poor solubility and poor bioavailability. For excessive large scale nanosuspensions production, high pressure homogenization and media milling technology have been viably used. Striking characteristics, similar to progress of dissolution velocity, enhanced saturation solubility, upgraded bioadhesivity, versatility in surface modification, and burden less postproduction processing have expanded the uses of nanosuspensions for different routes of administration.

\section{ACKNOWLEDGMENT}

The Authors are thankful to the Principal and Management of Vivekananda College of Pharmacy for providing all the necessary support and encouragement.

\section{AUTHORS CONTRIBUTIONS}

All the authors have contributed equally.

\section{CONFLICT OF INTERESTS}

The authors declare no conflict of interest.

\section{REFERENCES}

1. Chan VS. Nanomedicine: an unresolved regulatory issue. Regul Toxicol Pharmacol 2006;46:218-24.

2. Liversidge ME, Liversidge GG, Cooper ER. Nanosizing: a formulation approach for poor-water soluble compounds. Eur J Pharm Sci 2003;18:113-20.

3. Freitas RA. What is nanomedicine? Nanomedicine 2005;1:2-9.

4. Keck CM, Muller RH. Drug nanocrystals of poorly soluble drugs produced by high-pressure homogenisation. Eur J Pharm Biopharm 2006;62:3-16.

5. Shegokar R, Muller RH. Nanocrystals: industrially feasible multifunctional formulation technology for poorly soluble actives. Int J Pharm 2010;399:129-39.

6. Butler JM, Dressman JB. The developability classification system: application of biopharmaceutics concepts to formulation development. J Pharm Sci 2010;99:4940-54.

7. Moschwitzer JP. Drug nanocrystals in the commercial pharmaceutical development process. Int J Pharm 2013;453:142-56

8. Lipinski CA. Drug-like properties and the causes of poor solubility. J Pharmacol Toxicol Methods 2000;44:235-49.

9. Bevernage J, Brouwers J, Brewster ME, Augustijns P. Evaluation of gastrointestinal drug supersaturation and precipitation: strategies and issues. Int J Pharm 2013;453:25-35.
10. Dave RH, Shah DA, Patel PG. Development and evaluation of high loading oral dissolving film of aspirin and acetaminophen. J Pharm Sci Pharmacol 2014;1:112-22.

11. Cooper ER. Nanoparticles: a personal experience for formulating poorly water soluble drugs. J Controlled Release 2010;141:300-2.

12. Sigfridsson K, Lundqvist AJ, Strimfors M. Size reduction for improvement of oral absorption of the poorly soluble drug UG558 in rats during early development. Drug Dev Ind Pharm 2009;35:1479-86.

13. Liversidge GG, Conzentino P. Drug particle size reduction for decreasing gastric irritancy and enhancing absorption of naproxen in rats. Int J Pharm 1995;125:309-13.

14. Liversidge GG, Cundy KC. Particle size reduction for improvement of oral bioavailability of hydrophobic drugs: I. Absolute oral bioavailability of nanocrystallinedanazol in beagle dogs. Int J Pharm 1995;125:91-7.

15. Kesisoglou F, Panmai S, Wu Y. Nanosizing-oral formulation development and biopharmaceutical evaluation. Adv Drug Delivery Rev 2007;59:631-44.

16. Murdande SB, Pikal MJ, Shanker RM, Bogner RH. Solubility advantage of amorphous pharmaceuticals: I. A thermodynamic analysis. J Pharm Sci 2010;99:1254-64.

17. Murdande SB, Pikal MJ, Shanker RM, Bogner RH. Solubility advantage of amorphous pharmaceuticals: II. Application of quantitative thermodynamic relationships for prediction of solubility enhancement in structurally diverse insoluble pharmaceuticals. Pharm Res 2010;27:2704-14.

18. Liu G, Zhang D, Jiao Y, Guo H, Zheng D, Jia L, et al. In vitro and in vivo evaluation of riccardin $\mathrm{D}$ nanosuspensions with different particle size. Colloids Surf B 2013;102:620-6.

19. Detroja C, Chavhan S, Sawant K. Enhanced antihypertensive activity of candesartan cilexetil nanosuspension: formulation, characterization and pharmacodynamic study. Sci Pharm 2011;79:635-51.

20. Hecq J, Deleers M, Fanara D, Vranckx H, Amighi K. Preparation and characterization of nanocrystals for solubility and dissolution rate enhancement of nifedipine. Int J Pharm 2005;299:167-77.

21. Schmelzer JW, Schmelzer J. Kinetics of nucleation at increasing supersaturation. J Colloid Interface Sci 1999;215:345-55.

22. Shah KB, Patel PG, Khairuzzaman A, Bellantone RA. An improved method for the characterization of supersaturation and precipitation of poorly soluble drugs using pulsatile microdialysis (PMD). Int J Pharm 2014;468:64-74.

23. Cheow WS, Hadinoto K. Self-assembled amorphous drugpolyelectrolyte nanoparticle complex with enhanced dissolution rate and saturation solubility. J Colloid Interface Sci 2012;367:518-26.

24. Eerdenbrugh VB, Vermant J, Martens JA, Froyen L, Humbeeck JV, Mooter VDG, et al. Solubility increases associated with crystalline drug nanoparticles: methodologies and significance. Mol Pharm 2010;7:1858-70.

25. Williams HD, Trevaskis NL, Charman SA, Shanker RM, Charman WN, Pouton CW, et al. Strategies to address low drug solubility in discovery and development. Pharmacol Rev 2013;65:315499.

26. Sun B, Yeo Y. Nanocrystals for the parenteral delivery of poorly water-soluble drugs. Curr Opin Solid State Mater Sci 2012;16:295-301.

27. Barret ER. Nanosuspensions in drug delivery. Nat Rev Drug Discovery 2004;3:785-96.

28. Nanosuspension Systems. Hamamatsu Nano technology. Available from: http://www.hamanano.com/e/products/ C3/C3_1/. [Last accessed on 10 Jul 2019]

29. Raval JA, Patel JK, Patel MM. Nanosuspensions as particulate drug delivery systems. Pharm Rev 2006;4:5.

30. Prabhakar C, Krishna BK. A review on nanosuspensions in drug delivery. Int J Pharma Bio Sci 2011;2:549-58.

31. Rabinow BE. Nanosuspensions in drug delivery. Nat Rev Drug Discovery 2004;3:785-96.

32. Liu P, Rong X, Laru J. Nanosuspensions of poorly soluble drugs: preparation and development by wet milling. Int J Pharm 2011;411:215-22. 
33. Ibrahim HM, Ismail HR, Lila AEA. Formulation and optimization of ocular poly-D, L-lactic acid nano-drug delivery system of amphotericin-B using box behnken design. Int J Pharm Pharm Sci 2012;4:342-9.

34. Sutradhar KB, Khatun S, Luna IP. Increasing possibilities of nanosuspension. J Nanotechnol 2013. http://dx.doi.org/ $10.1155 / 2013 / 346581$.

35. Arole VM, Munde SV. Fabrication of nanomaterials by top-down and bottom-up approaches-an overview. J Adv Appl Sci Technol 2014;1:89-93.

36. Salazar J, Muller RH, Moschwitzer JP. Combinative particle size reduction $€$ technologies for the production of drug nanocrystals. J Pharm 2014;14. http://dx.doi.org/10.1155/ $2014 / 265754$.

37. Weber $\mathrm{U}$. The effect of grinding media performance on milling a water-based color pigment. Chem Eng Technol 2010;33:145663.

38. Singh SK, Srinivasan KK, Gowthamarajan K, Singare DS, Prakash D, Gaikwad NB. Investigation of preparation parameters of nanosuspension by top-down media milling to improve the dissolution of poorly water-soluble glyburide. Eur J Pharm Biopharm 2011;78:441-6.

39. Tashan E, Karakucuk A, Celebi N. Optimization and in vitro evaluation of ziprasidone nanosuspensions produced by a topdown approach. J Drug Delivery Sci Technol 2019;52:37-45.

40. Guo L, Kang L, Liu X, Lin X, Di D, Wu Y, et al. A novel nanosuspension of Andrographolide: preparation, characterization and passive liver target evaluation in rats. Eur J Pharm Sci 2017;104:13-22.

41. Shen C, Shen B, Liu X, Yuan H. Nanosuspensions based gel as the delivery system of nitrofurazone for enhanced dermal bioavailability. J Drug Delivery Sci Technol 2018;43:1-11.

42. Huang S, Zhang Q, Li H, Sun Y, Cheng G, Zou M, et al. Increased bioavailability of efonidipine hydrochloride nanosuspensions by the wet-milling method. Eur J Pharm Biopharm 2018;130:108-14.

43. Malamatari M, Taylor KMG, Malamataris S, Douroumis D, Kachrimanis K. Pharmaceutical nanocrystals: production by wet milling and applications. Drug Discovery Today 2018;23:534-47.

44. Reddy GA, Anilchowdary Y. Nanosuspension technology: a review. J Pharm Cosmetol 2012;2:47-52.

45. Wang H, Xiao Y, Wang H, Sang Z, Han X, Ren S, et al. Development of daidzein nanosuspensions: preparation, characterization, in vitro evaluation, and pharmacokinetic analysis. Int J Pharm 2019;566:67-76.

46. Karakucuk A, Teksin ZS, Eroglu H, Celebi N. Evaluation of improved oral bioavailability of ritonavir nanosuspension. Eur J Pharm Sci 2019;131:153-8.

47. Nagaraju P, Krishnachaithanya K, Srinivas VDN, Padma SVN. Nanosuspensions: promising drug delivery systems. Int J Pharm Sci Nanotechnol 2010;2:679-84.

48. Sumathi R, Tamizharasi S, Sivakumar T. Formulation and evaluation of polymeric nanosuspension of naringenin. Int $\mathrm{J}$ Appl Pharm 2017;9:60-70.

49. https://www.researchgate.net/figure/Schematic-diagram-ofthe-process-of-high-pressure-homogenization-21_fig.2_ 280444393. [Last accessed on 10 Jul 2019].

50. Kathpalia H, Juvekar S, Shidhaye S. Design and in vitro evaluation of atovaquone nanosuspension prepared by $\mathrm{ph}$ based and anti-solvent based precipitation method. J Colloid Interface Sci 2019;29:26-32.

51. Santos DAM, Carvalho FC, Teixeira DA, Azevedo DL, Barros WMD, Gremiao MPD. Computational and experimental approaches for the development of methotrexate nanosuspensions by bottom-up nanoprecipitation. Int J Pharm 2017;524:330-8.

52. Shariare MH, Altamimi MA, Marzan AL, Tabassum R, Jahan B, Reza HM, et al. In vitro dissolution and bioavailability study of furosemide nanosuspension prepared using a design of experiment (DoE). Saudi Pharm J 2018;27:96-105.
53. He S, Yang H, Zhang R, Li Y, Duan L. Preparation and in vitro-in vivo evaluation of teniposide nanosuspensions. Int J Pharm 2014;478:131-7.

54. Mishra B, Sahoo J, Dixit PK. Formulation and process optimization of naproxen nanosuspensions stabilized by hydroxyl propyl methylcellulose. Carbohydr Polym 2015;127:300-8.

55. Manishaanjane, Agrawal S, Khan A. Formulation and evaluation of nanosuspension of valsartan. Int J Curr Pharm Res 2018;10:68-74

56. Dekate S, Bhairy S, Hirlekar R. Preparation and characterization of oral nanosuspension loaded with curcumin. Int J Pharm Pharm Sci 2018;10:90-5.

57. Paun JS. Nanosuspension: an emerging trend for bioavailability enhancement of poorly soluble drugs. Asian J Pharm Tech 2012;2:157-68.

58. Vaghela A. Nanosuspension technology. Int J Universal Pharm Life Sci 2012;2:306-17.

59. Bhargavi R. Technical review of nanosuspensions. Int J Pharm Technol 2011;3:1503-11.

60. Verma KAK. Nanosuspensions: advantages and disadvantages. Indian J Novel Drug Delivery 2012;4:179-88.

61. Rao SK, Prasad T, Mohanta GP, Manna PK. An overview of statins as hypolipidemic drugs. Int J Pharm Sci Drug Res 2011;3:178-83.

62. Boedeker BH, Lojeski EW, Kline MD, Haynes DH. Ultra-long duration local anesthesia produced by injection of lecithincoated tetracaine microcrystals. J Clin Pharmacol 1994;34:699702.

63. Jia L, Wong H, Cerna C, Weitman SD. Effect of nanonization on absorption of 301029:Ex vivo and in vivo pharmacokinetic correlations determined by liquid chromatography/mass spectrometry. Pharm Res 2002;19:1091-6.

64. Liversidge ME. Formulation and antitumor activity evaluation of nanocrystalline suspensions of poorly soluble anticancer drugs. Pharm Res 1996;13:272-8.

65. Peters K, Leitzke S, Diederichs JE, Borner K, Hahn H, Muller RH, et al. Preparation of a clofazamine nanosuspension for intravenous use and evaluation of its therapeutic efficacy in murine Mycobacterium avium infection. J Antimicrob Chemother 2000;45:77-83.

66. Rainbow B, Kipp J, Papadopoulos P, Wong J, Glosson J, Gass J, et al. Itraconazole IV nanosuspension enhances efficacy through altered pharmacokinetics in the rat. Int J Pharm 2007;339:251-60.

67. Trejo HN, Kayser O, Steckel H, Muller RH. Characterization of nebulized bupravaquone nanosuspensions-effect of nebulization technology. J Drug Target 2005;13:499-507.

68. Mansour HM, Rhee YS, Wu X. Nanomedicine in pulmonary delivery. Int J Nanomed 2009;4:299-319.

69. Aher SS, Malsane ST, Saudagar RB. Nanosuspension: an overview. Int J Curr Pharm Res 2017;9:19-23.

70. Liang Y, Binner J. Effect of triblock copolymer non-ionic surfactants on the rheology of $3 \mathrm{~mol} \%$ yttria-stabilized zirconia nanosuspensions. Ceram Int 2008;34:293-7.

71. Kayser 0, Lemke A, Trejo HN. The impact of nanobiotechnology on the development of new drug delivery systems. Curr Pharm Biotechnol 2005;6:3-5.

72. Kayser 0. Nanosuspensions for the formulation of aphidicolin to improve drug targeting effects against leishmania infected macrophages. Int J Pharm 2000;196:253-6.

73. Scholer N, Krause K, Kayser O, Moller RH, Borner K, Hahn H, et al. Atovaquone nanosuspensions show excellent therapeutic effect in a new murine model of reactivated toxoplasmosis. Antimicrob Agents Chemother 2001;45:1771-9.

74. Du J, Li X, Zhao $\mathrm{H}$, Zhou Y, Wang L, Tian S, et al. Nanosuspensions of poorly water-soluble drugs prepared by bottom-up technologies. Int J Pharm 2015;495:738-49.

75. Lakshmi P, Kumar GA. Nano-suspension technology: a review. Int J Pharm Pharm Sci 2010;2:35-40. 